\title{
Thermography Study of the Pacient with Diabetic Foot Treated in a Rehabilitation Department
}

Adriana Sarah Nica, Gilda Mologhianu, Andreia Murgu, Florina Ojoga,Brindusa Sirghii, Svetlana llie, Ana Meila

University of Medicine and Pharmacy, Bucharest, Romania

Keywords

Rehabilitation, thermography, diabetic foot, pain, quality of life

\section{Introduction}

Diabetes is a severe pathology through vascular (micro and macroangiopathy) and neurological complications (neuropathy) with trophic complications that need surgical intervention. Diabetes has an important entity, the diabetic foot, and this type of consequence is analyzed using thermography, revealing secundary problems connected to the thermic adaptation system in the affected area of the body and they can be studied in the vasomotor and thermic context. Infrared thermography for the foot used to observe some local pathology is generally recognized, but the systemic and local context of the pacient with diabetic foot can be a new pathology in the thermography area research.

\section{Material}

We have studied 47 in-pacients with diabetic foot, using an EDP medical thermograph in standard evaluation conditions.

\section{Method}

We have applied a standard program of physical therapy. We recorded thermographies before and after each procedure. We have studied temperature gradients for single areas, we have compared the temperature gradients between the left and the right part of the body, and finally we have observed the therapy effect in time.

\section{Results}

The results were biostatistical transformed and interpreted and they underline different degrees of circulatory problems and the different types of answer and reaction in connection to the intensity of the main desease. 
http://dx.doi.org/10.21611/qirt.2008.03_10_17 